Homology, Homotopy and Applications, vol.12(2), 2010, pp.147-165

\title{
PSEUDO-COHOMOLOGY OF GENERAL EXTENSIONS
}

\author{
RAMJI LAL AND B.K. SHARMA \\ (communicated by Nicholas J. Kuhn)
}

\begin{abstract}
In this article, we associate new invariants called pseudohomology and pseudo-cohomology groups to right quasigroups and in turn to geometric spaces with natural quasigroup structures and to general extensions. It initiates a program to differentiate different types of general extensions.
\end{abstract}

\section{Introduction}

Eilenberg and MacLane $[\mathbf{3}, \mathbf{4}, \mathbf{5}]$ attached cohomology groups to Schreier's extensions (extension of a group by another group), studied it and realized its topological applications. Indeed, as an attempt to interpret higher cohomology groups, Eilenberg and MacLane [6] introduced the concept of loop prolongation and observed that cohomological cocycles measures the breach of associativity in the structure. With that, cohomology theory of groups took on extensive importance for application to almost all branches of mathematics. Further, Lafuente [10] realized a loop prolongation corresponding to a coupling $\chi: G \longrightarrow$ Out $A$ (in case the obstruction $\operatorname{obs}(G, A, \chi)=0$ ) which admits a parametrization like the one developed by Schreier for group extensions and also these extensions modulo equivalence of extensions are in bijective correspondence with $H^{2}(G, Z(A)) \times Z^{3}(G, Z(A))$. Baer [1] tried to study general extensions for the first time and he was confined to get relations between different types of general extensions of groups.

Apart from $[\mathbf{6}, \mathbf{1 0}]$ some attempts $[\mathbf{7}, \mathbf{1 7}]$ were made to study extensions of more general nonassociative structures (such as loops) by attaching certain invariants. Indeed, in $[\mathbf{7}, \mathbf{1 7}]$ the concept of loop modules and also loop cohomology is introduced and it is shown that the category of loops is a balanced category, whereas the category of commutative loops is not. In this article, we try to give cohomological interpretations to the group extensions by right loops and attach new groups called pseudo-cohomology and pseudo-homology groups to right quasigroups, to some geometric spaces with natural quasigroup structures and to general extensions. We also initiate a program to differentiate different types of general extensions, namely equivalent, almost equivalent and related extensions. They may be used further in some more branches of mathematics, for example, to fibrations with a unique path lifting property, where the fundamental group of the base space is a general extension

Received August 5, 2008, revised June 19, 2010; published on September 17, 2010.

2000 Mathematics Subject Classification: 20J05, 20 N05.

Key words and phrases: general extension, $c$-groupoid, right quasigroup, pseudo-cohomology.

This article is available at http://intlpress.com/HHA/v12/n2/a5

Copyright (C) 2010, International Press. Permission to copy for private use granted. 
of the fundamental group of the total space. In Section 9, we pose some problems on Dedekind-type groups in relation to low dimensional pseudo-cohomology of right transversals in groups.

\section{Acknowledgements}

We are grateful to the referee for his/her valuable comments and suggestions for improvement of the paper.

\section{Pseudo-chain complexes}

Definition 2.1. A pseudo-chain complex $(X, Y)$ of modules over a ring $R$ with identity is a family $\left\{\left(X_{n}, Y_{n}\right), d_{n} \mid n \in \mathbb{Z}\right\}$, where $Y_{n}$ is a submodule of $X_{n}$ and $d_{n}$ is a homomorphism from $X_{n}$ to $X_{n-1}$ such that $d_{n-1} d_{n}\left(Y_{n}\right)=\{0\}$ for all $n \in \mathbb{Z}$.

Thus every chain complex is a pseudo-chain complex with $X_{n}=Y_{n}$ for each $n$. The class $\mathcal{P C}$ of all pseudo-chain complexes of $R$-modules forms a category if we define a morphism (called a pseudo-chain transformation) between $(X, Y)$ and $\left(X^{\prime}, Y^{\prime}\right)$ to be a family $f=\left\{f_{n}: X_{n} \longrightarrow X_{n}^{\prime} \mid n \in \mathbb{Z}\right\}$ of homomorphisms such that:

(i) $f_{n}\left(Y_{n}\right) \subseteq Y_{n}^{\prime}$

(ii) $f_{n-1} d_{n}=d_{n}^{\prime} f_{n}$.

Let $(X, Y)$ be a pseudo-chain complex of $R$-modules. The submodule $Z_{n}=\operatorname{Ker} d_{n}$ of $X_{n}$ will be called the module of $n$-pseudo-cycles, and the submodule $B_{n}=d_{n+1}$ $\left(Y_{n+1}\right)$ of $X_{n}$ will be called the module of $n$-pseudo-boundaries of $(X, Y)$. The quotient module $Z_{n} / B_{n}$ denoted by $H_{n}(X, Y)$ is called the $n$-th pseudo-homology of $(X, Y)$. If $f$ is a pseudo-chain transformation from $(X, Y)$ to $\left(X^{\prime}, Y^{\prime}\right)$, then $f_{n}\left(Z_{n}\right) \subseteq Z_{n}^{\prime}$ and $f_{n}\left(d_{n+1}\left(Y_{n+1}\right)\right)=d_{n+1}^{\prime}\left(f_{n+1}\left(Y_{n+1}\right)\right) \subseteq d_{n+1}^{\prime}\left(Y_{n+1}^{\prime}\right)$. Thus $f$ induces a homomorphism $H_{n}(f)$ from $H_{n}(X, Y)$ to $H_{n}\left(X^{\prime}, Y^{\prime}\right)$. This gives us a covariant functor $H_{n}$ from $\mathcal{P C}$ to the category of $R$-modules. It can also be observed that the category $\mathcal{C}$ of chain complexes is a full subcategory of the category $\mathcal{P C}$ of pseudo-chain complexes.

Let $(X, Y)$ be a pseudo-chain complex of $R$-modules. Put

$$
F(X, Y)_{n}=Y_{n} \bigcap d_{n}^{-1}\left(Y_{n-1}\right) .
$$

Clearly, $d_{n}\left(F(X, Y)_{n}\right) \subseteq F(X, Y)_{n-1}$ and $F(X, Y)=\left\{F(X, Y)_{n},\left.d_{n}\right|_{F(X, Y)_{n}}\right\}$ is a chain complex which is a subpseudo-chain complex of $(X, Y)$. In addition,

$$
i=\left\{i_{n}: F(X, Y)_{n} \longrightarrow X_{n}\right\},
$$

where $i_{n}$ is the inclusion map for each $n$, is a pseudo-chain transformation. The functor $F$ thus obtained is a reflector functor from the category $\mathcal{P C}$ of pseudo-chain complexes to the subcategory $\mathcal{C}$ of chain complexes. Also, it follows that $H_{n}(i)$ is a monomorphism from $H_{n}(F(X, Y))$ to $H_{n}(X, Y)$ for each $n$. Thus $H_{n}(F(X, Y))$ can be naturally viewed as a submodule of $H_{n}(X, Y)$.

We have another functor $G$ from $\mathcal{P C}$ to $\mathcal{C}$ given by $G(X, Y)=\left\{Y_{n}+Z_{n},\left.d_{n}\right|_{Y_{n}+Z_{n}}\right\}$. One observes that $H_{n}(X, Y)=H_{n}(G(X, Y))$ for each $n$. 
Let $(X, Y)$ and $(K, L)$ be two pseudo-chain complexes of $R$-modules, where $R$ is a commutative ring with identity. Define $(X \otimes K)_{n}=\bigoplus_{p+q=n} X_{p} \otimes K_{q}$ and $(Y \otimes L)_{n}$ to be the submodule of $(X \otimes K)_{n}$ generated by elements $x \otimes y$, where

(i) $x$ is a $p$-pseudo-cycle and $y \in L_{q}$,

(ii) $x \in Y_{p}$ and $y$ is a $q$-pseudo-cycle in $K_{q}$, or

(iii) $x \in Y_{p}$ and $y \in L_{q}$,

where $p+q=n$.

Define

$$
\overline{d_{n}}(x \otimes y)=d_{p}(x) \otimes y+(-1)^{p} x \otimes d_{q}(y), p+q=n .
$$

Then $\left\{\left((X \otimes K)_{n},(Y \otimes L)_{n}\right), \overline{d_{n}}\right\}$ is a pseudo-chain complex called the tensor product of $(X, Y)$ and $(K, L)$ and is denoted by $(X, Y) \otimes(K, L)$.

A natural problem here is to see the relationship between the pseudo-homologies of $(X, Y),(K, L)$ and their tensor product. A partial solution to the problem can be given with the help of the Künneth formula.

We have a homomorphism $\mu$ from

$$
H_{m}(X, Y) \otimes H_{q}(K, L) \quad \text { to } \quad H_{m+q}((X, Y) \otimes(K, L))
$$

given by $\mu([x] \otimes[y])=[x \otimes y]$. This gives us a homomorphism, again denoted by $\mu$, from

$$
\bigoplus_{m+q-n} H_{m}(X, Y) \otimes H_{q}(K, L)
$$

to $H_{n}((X, Y) \otimes(K, L))$. This homomorphism is called the pseudo-homology product.

Proposition 2.2. Let $(X, Y)$ be a pseudo-chain complex such that the module $Z_{n}$ of n-pseudo-cycles and the module $B_{n}$ of n-pseudo-boundaries are flat modules for each $n$. Then for any pseudo-chain complex $(K, L)$, the pseudo-homology product is a monomorphism.

Proof. Under the hypothesis of the proposition, the Künneth formula [15, Theorem 10.2 ] can be applied to the complex $G(X, Y)$ and we have an exact sequence

$$
\begin{aligned}
& 0 \longrightarrow \bigoplus_{m+q=n} H_{m}(X, Y) \otimes H_{q}(K, L) \stackrel{P}{\longrightarrow} H_{n}(G(X, Y) \otimes G(K, L)) \\
& \longrightarrow \bigoplus_{m+q=n-1} \operatorname{Tor}\left(H_{m}(X, Y), H_{q}(K, L)\right) \longrightarrow 0,
\end{aligned}
$$

where $P$ is the homology product. We have a homomorphism $\phi$ from the image of the pseudo-homology product $\mu$ to $H_{n}(G(X, Y) \otimes G(K, L))$ defined by $\phi([x \otimes k])=$ $[x \otimes k]$, where $x$ is a $m$-pseudo-cycle in $(X, Y)$ and $k$ is a $q$-pseudo-cycle in $(K, L)$, $m+q=n$. The result follows if we note that $\phi \circ \mu=P$.

Corollary 2.3. If $H_{n}(X, Y)$ and $C_{n}$ are projective modules for each $n$, then

$$
H_{n}(G(X, Y) \otimes G(K, L))
$$

is a submodule of $H_{n}((X, Y) \otimes(K, L))$.

It may be interesting to describe the cokernel of the pseudo-homology product under the hypothesis of Proposition 2.2. 


\section{Some preliminaries}

Everything in this section has been taken from $[\mathbf{1 1}]$.

Definition 3.1 ([11, Definition 2.1]). A quadruple $(S, H, \sigma, f)$, where $S$ is a groupoid with identity $e, H$ a group which acts on $S$ from right through a given action $\theta, \sigma$ a map from $S$ to $H^{H}$ (the set of all maps from $H$ to $H$ ) and $f$ a map from $S \times S$ to $H$, is called a $c$-groupoid if it satisfies the following conditions:

$\left(C_{1}\right)[x \circ y=y] \Longrightarrow x=e$,

$\left(C_{2}\right)$ For each $x \in S$, there exists $x^{\prime} \in S$ such that $x^{\prime} \circ x=e$,

$\left(C_{3}\right) \sigma_{e}=I_{H}$, the identity map on $H$, where $\sigma_{x}$ denotes the image of $x$ under the $\operatorname{map} \sigma$,

$\left(C_{4}\right) f(x, e)=f(e, x)=1$, the identity of $H$,

$\left(C_{5}\right) \sigma_{x}\left(h_{1} h_{2}\right)=\sigma_{x}\left(h_{1}\right) \sigma_{x \theta h_{1}}\left(h_{2}\right)$,

$\left(C_{6}\right)(x \circ y) \circ z=x \theta f(y, z) \circ(y \circ z)$,

$\left(C_{7}\right)(x \circ y) \theta h=x \theta \sigma_{y}(h) \circ(y \theta h)$,

$\left(C_{8}\right) f(x, y) f(x \circ y, z)=\sigma_{x}(f(y, z)) f(x \theta f(y, z), y \circ z)$,

$\left(C_{9}\right) f(x, y) \sigma_{x \circ y}(h)=\sigma_{x}\left(\sigma_{y}(h)\right) f\left(x \theta \sigma_{y}(h), y \theta h\right)$, where $x, y, z \in S$ and $h_{1}, h_{2}, h \in H$.

The following identities are immediate in a $c$-groupoid $(S, H, \sigma, f)$ :

(i) $\sigma_{x}(1)=1$ for all $x \in S$.

(ii) $e \theta h=h$ for all $h \in H$.

Given a right transversal $S$ of a subgroup $H$ of a group $G$, we have the binary operation $\circ$, the action $\theta$ of $H$ on $S$, the maps $f$ and $\sigma$ defined by the equations $x \cdot y=f(x, y)(x \circ y)$ and $x \cdot h=\sigma_{x}(h)(x \theta h)$ such that $(S, H, \sigma, f)$ is a $c$-groupoid. Conversely, we have the following result:

Theorem 3.2 ([11, Theorem 2.2]). Let $(S, H, \sigma, f)$ be a c-groupoid. Then there exists a group $G$ which contains $H$ as a subgroup and $S$ as a right transversal of $H$ in $G$ such that the corresponding c-groupoid is $(S, H, \sigma, f)$.

As a consequence we obtain the following corollary:

Corollary 3.3 ([11, Corollary 2.4]). If $(S, H, \sigma, f)$ is a c-groupoid, then $(S, \circ)$ is a right loop (right quasigroup with identity).

Conversely, let $(S, \circ)$ be a right quasigroup with identity $e$. Let $y, z \in S$ and denote the unique solution of the equation

$$
X \circ(y \circ z)=(x \circ y) \circ z
$$

by $f^{s}(y, z)(x)$. This gives us a map $f^{s}(y, z)$ from $S$ to $S$, which can easily be seen to be a member of $\operatorname{Sym}(S)$, the symmetric group on $S$. Indeed, $f^{s}(y, z)$ fixes $e$ and so it can be considered as a member of $\operatorname{Sym}(S \backslash\{e\})$. This also gives us a map $f^{s}$ from $S \times S$ to $\operatorname{Sym}(S \backslash\{e\})$. The group $\operatorname{Sym}(S)$ and so also $\operatorname{Sym}(S \backslash\{e\})$ acts on $S$ from the right in a natural manner, if we adopt the convention 


$$
(r \cdot s)(x)=s(r(x)) ; r, s \in \operatorname{Sym}(S)
$$

for the product in $\operatorname{Sym}(S)$. We denote this action by $\theta^{s}$. Next, let $x \in S$ and $h \in$ $\operatorname{Sym}(S \backslash\{e\})$. Again $\sigma_{y}^{s}(h)(x)$ denotes the unique solution of the equation

$$
X \circ\left(y \theta^{s} h\right)=(x \circ y) \theta^{s} h .
$$

It follows again that the map $\sigma_{y}^{s}(h)$ from $S$ to $S$ thus obtained is a member of $\operatorname{Sym}(S)$ fixing $e$. This gives us the map $\sigma^{s}$ from $S$ to $H^{H}$, where $H=\operatorname{Sym}(S \backslash\{e\})$. It can be verified (see [11]) that $\left(S, \operatorname{Sym}(S \backslash\{e\}), \sigma^{s}, f^{s}\right)$ is a $c$-groupoid. The corresponding extension of $\operatorname{Sym}(S \backslash\{e\})$ denoted by $G^{S}$ is called the general extension of $\operatorname{Sym}(S \backslash\{e\})$ by $S$, which is universal in a certain sense. The subgroup $G_{S}$ of $\operatorname{Sym}(S \backslash\{e\})$ generated by $\left\{f^{s}(y, z) \mid y, z \in S\right\}$, which is indeed the intersection of $\operatorname{Sym}(S \backslash\{e\})$ with the subgroup of $G^{S}$ generated by $S$, is called the group torsion also called the inner mapping group of $S$. Clearly $f^{s}$ is a map from $S \times S$ to $G_{S}$. It also follows that $\sigma^{s}$ gives a map from $S$ to $G_{S}^{G_{S}}$ so that $\left(S, G_{S}, \sigma^{s}, f^{s}\right)$ is a $c$-groupoid. The corresponding group extension $G_{S} S$ is called the group extension determined by $S$. Note that this is the smallest group in which $S$ can be realized as a transversal.

Consider the subgroup $\langle S\rangle$ of a group $G$ generated by a right transversal $S$ of a subgroup $H$ in $G$. Let $H_{S}=\langle S\rangle \cap H$. Then $H_{S}=\left\langle\left\{x y(x \circ y)^{-1} \mid x, y \in S\right\}\right\rangle$, where 'o' is the induced binary operation on $S$ (as discussed earlier) and $H_{S} S=\langle S\rangle$. Identifying $S$ with the set $G /{ }^{r} H$ of right cosets of $H$ in $G$, we obtain the permutation representation $\phi: G \longrightarrow \operatorname{Sym}(S)(\{\phi(g)(x)\}=S \cap H x g ; g \in G, x \in S)$. The group $G_{S}$ described earlier is precisely $\phi\left(H_{S}\right)$.

Let $H^{S}$ be the smallest normal subgroup of $\langle S\rangle=H_{S} S$ which contains $H_{S}$. Let $M(S)$ denote the quotient group $H_{S} S / H^{S} \cong G_{S} S / \phi\left(H^{S}\right)$. Clearly, $M(S)$ depends only on the right quasigroup structure on $S$ and has a presentation $\langle S ; R\rangle$, where $R=\left\{x y(x \circ y)^{-1} \mid x, y \in S\right\}$ is the set of relators of the presentation, with ' $\circ$ ' being the binary operation induced on $S$. There is a homomorphism $\alpha: S \longrightarrow M(S)$ from the right quasigroup $(S, \circ)$ to $M(S)$ given by $\alpha(x)=H^{s} x$ such that given any group $K$ and a homomorphism $\beta: S \longrightarrow K$, there is a unique homomorphism $\eta: M(S) \longrightarrow K$ such that $\beta=\eta \circ \alpha$.

Let $\left(S^{1}, K^{1}, \sigma^{1}, f^{1}\right)$ and $\left(S^{2}, K^{2}, \sigma^{2}, f^{2}\right)$ be two $c$-groupoids. A triple $(p, q, g)$, where $p: S^{1} \longrightarrow S^{2}$ is a map, $q: K^{1} \longrightarrow K^{2}$ a homomorphism and $g: S^{1} \longrightarrow K^{2}$ a map such that $g(e)=1$, is called a c-homomorphism from $\left(S^{1}, K^{1}, \sigma^{1}, f^{1}\right)$ to $\left(S^{2}, K^{2}, \sigma^{2}, f^{2}\right)$ if:

$\left(C H_{1}\right) \quad p(x \circ y)=(p(x) \theta g(y)) \circ p(y)$,

$\left(\mathrm{CH}_{2}\right) q\left(f^{1}(x, y)\right) g(x \circ y)=g(x) \sigma_{p(x)}^{2}(g(y)) f^{2}(p(x) \theta g(y), p(y))$,

$\left(\mathrm{CH}_{3}\right) \quad p(x \theta k)=p(x) \theta q(k)$,

$\left(\mathrm{CH}_{4}\right) \quad q\left(\sigma_{x}^{1}(k)\right)=g(x) \sigma_{p(x)}^{2}(q(k))(g(x \theta k))^{-1}$,

for all $x, y \in S^{1}$ and $k \in K^{1}$.

Let $\left(p_{1}, q_{1}, g_{1}\right)$ be a $c$-homomorphism from $\left(S^{1}, K^{1}, \sigma^{1}, f^{1}\right)$ to $\left(S^{2}, K^{2}, \sigma^{2}, f^{2}\right)$ and $\left(p_{2}, q_{2}, g_{2}\right)$ a $c$-homomorphism from $\left(S^{2}, K^{2}, \sigma^{2}, f^{2}\right)$ to $\left(S^{3}, K^{3}, \sigma^{3}, f^{3}\right)$. Then $(p, q, g)$, where $p=p_{2} \circ p_{1}, q=q_{2} \circ q_{1}$ and $g(x)=q_{2}\left(g_{1}(x)\right) \cdot g_{2}\left(p_{1}(x)\right)$ for all $x \in S^{1}$, is a $c$-homomorphism from $\left(S^{1}, K^{1}, \sigma^{1}, f^{1}\right)$ to $\left(S^{3}, K^{3}, \sigma^{3}, f^{3}\right)$ called the composite of $\left(p_{1}, q_{1}, g_{1}\right)$ and $\left(p_{2}, q_{2}, g_{2}\right)$. This gives us a category $\mathcal{C G}$ of $c$-groupoids which can be 
seen $([\mathbf{1 1}])$ to be equivalent to the category $\mathcal{E}$ (the objects of $\mathcal{E}$ are pairs $(G, H)$ of groups, where $H$ is a subgroup of $G$ and a morphism $f$ from $\left(G_{1}, K_{1}\right)$ to $\left(G_{2}, K_{2}\right)$ is a group homomorphism from $G_{1}$ to $G_{2}$ such that $f\left(K_{1}\right) \subseteq K_{2}$ ) of general extensions.

\section{Pseudo-cohomology of $c$-groupoids and right quasigroups}

Definition 4.1. Let $(S, K, \sigma, f)$ be a $c$-groupoid. A sequence $\left(x_{1}, x_{2}, \ldots, x_{n}, \ldots\right)$ of elements of $S$ is called a $\sigma$-sequence in $(S, K, \sigma, f)$ if $\sigma_{x_{i}}\left(f\left(x_{i+1}, x_{i+2}\right)\right)=1$ for all $i>1$.

For a sequence $\left(x_{1}, x_{2}, \ldots, x_{n}, \ldots\right)$ in $S$, we define $A_{i_{1} i_{2} \cdots i_{r}}\left(x_{1}, x_{2}, \ldots, x_{n}, \ldots\right)$ by induction on $r$ as follows: Define

$A_{i_{1}}\left(x_{1}, x_{2}, \ldots, x_{n}, \ldots\right)=\left(x_{1}, \ldots, x_{i_{1}-1}, x_{i_{1}} \theta f\left(x_{i_{1}+1}, x_{i_{1}+2}\right), x_{i_{1}+1} \circ x_{i_{1}+2}, x_{i_{1}+3}, \ldots\right)$.

Supposing that $A_{i_{1} \cdots i_{r}}\left(x_{1}, x_{2}, \ldots, x_{n}, \ldots\right)$ has already been defined and is

$$
\left(y_{1}, y_{2}, \ldots, y_{n}, \ldots\right)
$$

we define $A_{i_{1} i_{2} \cdots i_{r+1}}\left(x_{1}, x_{2}, \ldots, x_{n}, \ldots\right)$ to be $A_{i_{r+1}}\left(y_{1}, y_{2}, \ldots, y_{n}, \ldots\right)$.

Definition 4.2. A $\sigma$-sequence $\left(x_{1}, x_{2}, \ldots, x_{n}, \ldots\right)$ will be called an $\boldsymbol{A}$-sequence in $(S, K, \sigma, f)$ if each $A_{i_{1} i_{2} \cdots i_{r}}\left(x_{1}, x_{2}, \ldots, x_{n}, \ldots\right)$ is a $\sigma$-sequence.

Remark 4.3. If $\left(x_{1}, x_{2}, \ldots, x_{n}, \ldots\right)$ is an $\mathbf{A}$-sequence in $(S, K, \sigma, f)$, then it is also an A-sequence in $\left(S, G_{S}, \sigma^{s}, f^{s}\right)$ as well as in $\left(S, \operatorname{Sym}(S \backslash\{e\}), \sigma^{s}, f^{s}\right)$.

Let $M$ be an abelian group and $C^{n}$ be the set of all maps from

$$
S^{n}=\underbrace{S \times S \times \cdots \times S}_{n \text { copies }}
$$

to $M$ such that $g\left(x_{1}, x_{2}, \ldots, x_{n}\right)=0$ if some $x_{i}=e$. By convention, $S^{0}=\{e\}$ and thus $C^{0}=\{0\}$. Then $C^{n}$ is an abelian group under the obvious addition. The subset

$$
\begin{aligned}
D^{n}=\left\{g \in C^{n} \mid\right. & g\left(x_{1}, x_{2}, \ldots, x_{n-1}, x_{n} \theta f\left(x_{n+1}, x_{n+2}\right)\right)=g\left(x_{1}, x_{2}, \ldots, x_{n}\right) \\
& \text { whenever } \left.\left(x_{1}, x_{2}, \ldots, x_{n}, x_{n+1}, x_{n+2}, e, e, \ldots\right) \text { is an } \boldsymbol{A} \text {-sequence }\right\}
\end{aligned}
$$

is a subgroup of $C^{n}$.

Define a map $\delta^{n}: C^{n} \longrightarrow C^{n+1}$ as follows: Let $g \in C^{n}$. Then

$$
\begin{aligned}
& \delta^{n} g\left(x_{1}, x_{2}, \ldots, x_{n+1}\right) \\
& = \begin{cases}0, & \text { if }\left(x_{1}, x_{2}, \ldots, x_{n+1}, e, e, \ldots\right) \text { is not an A-sequence, } \\
g\left(x_{2}, x_{3}, \ldots, x_{n+1}\right) \\
+\sum_{t=1}^{n}(-1)^{t} g\left(x_{1}, x_{2}, \ldots, x_{t-2}, x_{t-1} \theta f\left(x_{t}, x_{t+1}\right), x_{t} \circ x_{t+1}, x_{t+2}, \ldots, x_{n+1}\right) \\
+(-1)^{n+1} g\left(x_{1}, x_{2}, \ldots, x_{n}\right), & \text { otherwise. }\end{cases}
\end{aligned}
$$

Theorem 4.4. $\left\{\left(C^{n}, D^{n}\right), \delta^{n}\right\}$ is a pseudo-cochain complex.

We need the following lemma for proof of the Theorem 4.4.

Lemma 4.5. Let $(S, K, \sigma, f)$ be a c-groupoid. Suppose that $\sigma_{x}(f(y, z))=1$. Then: 
(i) $(u \circ x) \theta f(y, z)=u \circ x \theta f(y, z)$,

(ii) $f(x, y) f(x \circ y, z)=f(x \theta f(y, z), y \circ z)$.

Further, if $x, y, u, v$ are elements of $S$ such that $\sigma_{y}(f(u, v))=1$, then $\sigma_{x \circ y}(f(u, v))$ $=1$ if and only if $f(x, y)=f(x, y \theta f(u, v))$.

The proof of the lemma follows from $C_{7}, C_{8}$ and $C_{9}$.

Proof of Theorem 4.4. It is sufficient to show that $(\delta \circ \delta)\left(g\left(x_{1}, x_{2}, \ldots, x_{n+1}\right)\right)=g\left(x_{1}, x_{2}, \ldots, x_{n-1} \theta f\left(x_{n}, x_{n+1}\right)\right)-g\left(x_{1}, x_{2}, \ldots, x_{n-1}\right)$, whenever $\left(x_{1}, x_{2}, \ldots, x_{n+1}, e, e, \ldots\right)$ is an $\mathbf{A}$-sequence.

Suppose that $\left(x_{1}, x_{2}, \ldots, x_{n+1}, e, e, \ldots\right)$ is an $\mathbf{A}$-sequence. Then each

$$
A_{i}\left(x_{1}, x_{2}, \ldots, x_{n+1}, e, e, \ldots\right)
$$

is an $\mathbf{A}$-sequence and

$$
(\delta \circ \delta)\left(g\left(x_{1}, x_{2}, \ldots, x_{n+1}\right)\right)
$$

is a sum of $n+2$ terms $u_{i}(1 \leqslant i \leqslant n+2)$ and each $u_{i}$ is the sum of $n+1$ terms $u_{i, j}(1 \leqslant j \leqslant n+1)$. Clearly,

$$
u_{n+1, n+1}=g\left(x_{1}, x_{2}, \ldots, x_{n-1} \theta f\left(x_{n}, x_{n+1}\right)\right)
$$

and

$$
u_{n+2, n+1}=-g\left(x_{1}, x_{2}, \ldots, x_{n-1}\right) .
$$

For $j \neq n+1$,

$$
u_{n+2, j}=(-1)^{n+1}(-1)^{j-1} g\left(x_{1}, x_{2}, \ldots, x_{j-2} \theta f\left(x_{j-1}, x_{j}\right), x_{j-1} \circ x_{j}, x_{j+1}, \ldots, x_{n}\right) .
$$

Now suppose that $i \neq n+2$. Then

$$
\begin{aligned}
&(-1)^{i-1}(-1)^{j-1} u_{i, j} \\
&= g\left(x_{1}, x_{2}, \ldots, x_{j-2} \theta f\left(x_{j-1}, x_{j}\right), x_{j-1} \circ x_{j}, x_{j+1}, \ldots, x_{i-2} \theta f\left(x_{i-1}, x_{i}\right),\right. \\
&\left.x_{i-1} \circ x_{i}, x_{i+1}, \ldots, x_{n+1}\right), j<i-2 \\
&= g\left(x_{1}, x_{2}, \ldots, x_{i-4} \theta f\left(x_{i-3}, x_{i-2} \theta f\left(x_{i-1}, x_{i}\right)\right), x_{i-3} \circ x_{i-2} \theta f\left(x_{i-1}, x_{i}\right),\right. \\
&\left.x_{i-1} \circ x_{i}, x_{i+1}, \ldots, x_{n+1}\right), j=i-2 \\
&= g\left(x_{1}, x_{2}, \ldots, x_{i-3} \theta f\left(x_{i-2} \theta f\left(x_{i-1}, x_{i}\right), x_{i-1} \circ x_{i}\right), \ldots,\right. \\
&\left.x_{i-2} \theta f\left(x_{i-1}, x_{i}\right) \circ\left(x_{i-1} \circ x_{i}\right), x_{i+1}, \ldots, x_{n+1}\right), j=i-1 \\
&= g\left(x_{1}, x_{2}, \ldots, x_{i-2} \theta f\left(x_{i-1}, x_{i}\right) f\left(x_{i-1} \circ x_{i}, x_{i+1}\right),\left(x_{i-1} \circ x_{i}\right) \circ x_{i+1},\right. \\
&\left.x_{i+2}, \ldots, x_{n+1}\right), j=i, i \neq n+1 \\
&= g\left(x_{1}, x_{2}, \ldots, x_{i-2} \theta f\left(x_{i-1}, x_{i}\right),\left(x_{i-1} \circ x_{i}\right) \theta f\left(x_{i+1}, x_{i+2}\right), x_{i+1} \circ x_{i+2},\right. \\
&\left.x_{i+3}, \ldots, x_{n+1}\right), j=i+1 \\
&= g\left(x_{1}, x_{2}, \ldots, x_{i-2} \theta f\left(x_{i-1}, x_{i}\right), x_{i-1} \circ x_{i}, \ldots, x_{j-1} \theta f\left(x_{j}, x_{j+1}\right),\right. \\
&\left.x_{j} \circ x_{j+1}, x_{j+2}, \ldots, x_{n+1}\right), n+1 \neq j>i+1 \\
&= g\left(x_{1}, x_{2}, \ldots, x_{i-1} \theta f\left(x_{i-1}, x_{i}\right), x_{i-1} \circ x_{i}, \ldots, x_{n}\right), i \neq n+1, \\
& j=n+1 .
\end{aligned}
$$


From (1) and (8), it follows that $u_{n+2, j}=-u_{j, n+1}$ for $j \neq n+1$. If $i \neq n+2$, then from (2) and (7), we have $-u_{i, j}=u_{j, i-1}$, where $j<i-2$, equations (3), (6) and Lemma 4.5 yield that $u_{i, i-2}=-u_{i-2, i-1}$. Further, $u_{i, i-1}=-u_{i-1, i-1}$, which follows from equations (4), (5) and Lemma 4.5. Thus $u_{i, j}=-u_{j+1, i}$, whenever $i \neq n+1$ or $j \neq n+1$. The result now follows.

The pseudo-cochain complex $\left\{\left(C^{n}, D^{n}\right), \delta^{n}\right\}$ thus obtained will be denoted by

$$
P^{*}(S, K, \sigma, f, M)
$$

and its pseudo-homology will be called the pseudo-cohomology of $(S, K, \sigma, f)$ with coefficients in $M$. The $n$-th pseudo-cohomology of $(S, K, \sigma, f)$ will be denoted by $H^{n}(S, K, \sigma, f, M)$.

Given any right quasigroup $(S, \circ)$, the $n$-th pseudo-cohomology of $\left(S, G_{S}, \sigma^{s}, f^{s}\right)$ with coefficients in an abelian group $M$ will be denoted by $H^{n}(S, M)$ and called the $n$-th pseudo-cohomology of the right quasigroup $(S, \circ)$ with coefficients in $M$. It may be observed that $H^{n}\left(S, G_{S}, \sigma^{s}, f^{s}, M\right)=H^{n}\left(S, \operatorname{Sym}(S \backslash\{e\}), \sigma^{s}, f^{s}, M\right)$. If $S$ is a group, then $H^{n}(S, M)$ is the usual cohomology of the group $S$ with coefficients in the trivial $S$-module $M$. A $c$-homomorphism $(p, q, g)$ from $(S, K, \sigma, f)$ to $\left(S^{1}, K^{1}, \sigma^{1}, f^{1}\right)$ will be called a regular $c$-homomorphism if $q$ is injective and $g(x)=e$ for all $x \in S$. In this case,

(i) $p(x \circ y)=p(x) \circ p(y)$,

(ii) $q(f(x, y))=f^{1}(p(x), p(y))$,

(iii) $p(x \theta k)=p(x) \theta q(k)$,

(iv) $q\left(\sigma_{x}(k)\right)=\sigma_{p(x)}(q(k))$,

for all $x, y \in S$ and $k \in K$. A regular $c$-homomorphism $(p, q, g)$ will be denoted by $(p, q)$.

We observe that if $(p, q)$ is a regular $c$-homomorphism from

$$
(S, K, \sigma, f) \quad \text { to } \quad\left(S^{1}, K^{1}, \sigma^{1}, f^{1}\right),
$$

then $\left(x_{1}, x_{2}, \ldots, x_{n}, \ldots\right)$ is an $\mathbf{A}$-sequence in $(S, K, \sigma, f)$ if and only if

$$
\left(p\left(x_{1}\right), p\left(x_{2}\right), \ldots, p\left(x_{n}\right) \ldots\right)
$$

is an A-sequence in $\left(S^{1}, K^{1}, \sigma^{1}, f^{1}\right)$. For $\sigma_{x_{i}}\left(f\left(x_{i}, x_{i+1}\right)\right)=1$ if and only if

$$
\sigma_{p\left(x_{i}\right)}\left(f_{1}\left(p\left(x_{i}\right), p\left(x_{i+1}\right)\right)\right)=q\left(\sigma_{x_{i}} f\left(x_{i}, x_{i+1}\right)\right)=1 .
$$

Let $\mathcal{S}_{K}$ be the category with objects the $c$-groupoids $(S, K, \sigma, f)$ (for fixed $K$ ) and with morphisms the regular $c$-homomorphisms. Let $(p, q)$ be a regular $c$-homomorphism from $(S, K, \sigma, f)$ to $\left(S^{1}, K^{1}, \sigma^{1}, f^{1}\right)$. Let

$$
P^{*}(S, K, \sigma, f, M)=\left\{\left(C_{1}^{n}, D_{1}^{n}\right), \delta_{1}^{n}\right\}
$$

and

$$
P^{*}\left(S^{1}, K^{1}, \sigma^{1}, f^{1}, M\right)=\left\{\left(C_{2}^{n}, D_{2}^{n}\right), \delta_{2}{ }^{n}\right\} .
$$

Let $\phi \in C_{2}^{n}$. Define

$$
(p, q)^{*}(\phi)\left(x_{1}, x_{2}, \ldots, x_{n}\right)=\phi\left(p\left(x_{1}\right), p\left(x_{2}\right), \ldots, p\left(x_{n}\right)\right) .
$$


One can easily check that $(p, q)^{*}\left(D_{2}^{n}\right) \subseteq D_{1}^{n}$ and $\delta_{1}(p, q)^{*}=(p, q)^{*} \delta_{2}$. Thus $(p, q)$ induces a pseudo-cochain transformation from

$$
P^{*}\left(S^{1}, K^{1}, \sigma^{1}, f^{1}, M\right) \text { to } P^{*}(S, K, \sigma, f, M) .
$$

This gives us a contravariant functor $P^{*}$ from $\mathcal{S}_{K}$ to the category of pseudo-complexes of abelian groups and, in turn, allows us to say that $H^{n}$ for each $n$ is a contravariant functor from $\mathcal{S}_{K}$ to the category of abelian groups.

\section{Pseudo-cohomology of hyperbolic and spherical spaces with natural quasigroup structures}

The positive special Lorentz group

$$
\operatorname{PSO}(n, 1)=\left\{A \in \operatorname{GL}(n+1, \mathbb{R}) \mid A^{t} J A=J, \operatorname{Det} A=1 \text { and }\left\langle e_{n+1} A, e_{n+1}\right\rangle>0\right\},
$$

where $J$ is the matrix given by

$$
J=\left(\begin{array}{cc}
I_{n} & 0_{n \times 1} \\
0_{1 \times n} & -1
\end{array}\right)
$$

acts transitively from the right on the hyperbolic $n$-space

$$
\mathbb{H}^{n}=\left\{\bar{x}=\left(x_{1}, \ldots, x_{n}, x_{n+1}\right) \in \mathbb{R}^{n+1} \mid\left(\sum_{i=1}^{n}{x_{i}}^{2}\right)-x_{n+1}{ }^{2}=-1, x_{n+1}>0\right\},
$$

and the isotropy group of the action at $e_{n+1}=(0,0, \ldots, 0,1)$ is $\mathrm{SO}(n) \times \mathrm{SO}(1)$. Thus we can identify $\mathbb{H}^{n}$ with the set of right cosets of $\mathrm{SO}(n) \times \mathrm{SO}(1)$ in $\operatorname{PSO}(n, 1)$. The polar decomposition ensures that every matrix $A \in \operatorname{PSO}(n, 1)$ can be expressed uniquely as $A=U K$, where $U \in \mathrm{SO}(n) \times \mathrm{SO}(1)$ and $K \in S=\mathrm{PSO}(n, 1) \cap \mathcal{H}$, where $\mathcal{H}$ is the set of positive definite real $(n+1) \times(n+1)$-symmetric matrices. Thus $S$ can be taken as a right (indeed, it is also a left) transversal of $\mathrm{SO}(n) \times \mathrm{SO}(1)$ in $\operatorname{PSO}(n, 1)$, which can be identified with the hyperbolic space $\mathbb{H}^{n}$ through the map $\phi$ from $S$ to $\mathbb{H}^{n}$ given by $\phi(K)=e_{n+1} K$, for all $K \in S$. Indeed, this identification also respects the respective topologies. The right quasigroup structure ' $O$ ' on $S$ and the map $f: S \times S \longrightarrow \mathrm{SO}(n) \times \mathrm{SO}(1)$ is given by the polar decomposition $K L=f(K, L)(K \circ L)$, where $K, L \in S, K \circ L \in S$ and $f(K, L) \in \mathrm{SO}(n) \times \mathrm{SO}(1)$. This means that $K \circ L$ is the positive square root of $L K^{2} L$. Thus $f(K, L)$ is identity of $\mathrm{SO}(n) \times \mathrm{SO}(1)$ if and only if $K L=L K$. Since $K U=U\left(U^{-1} K U\right)$ is the polar decomposition of $K U, K \in S$ and $U \in \mathrm{SO}(n) \times \mathrm{SO}(1)$, the map $\sigma$ and the action $\theta$ (see the paragraph preceding Theorem 3.2) is given by $\sigma_{K}(U)=U$ and $K \theta U=U^{-1} K U$. Thus

Proposition 5.1. The group torsion $G_{S} \cong \mathrm{SO}(n) \times \mathrm{SO}(1)$ and for $P, K, L$ in $S$,

$$
\sigma_{P}(f(K, L))=f(K, L)=I
$$

if and only if $K L=L K$. This is true if and only if $K \circ L=L \circ K$.

Corollary 5.2. A sequence $\left(K_{1}, K_{2}, \ldots, K_{n}, I, I, \ldots\right)$ in $S$ is an A-sequence if and only if $K_{i} K_{j}=K_{j} K_{i}$ for all $i, j \geqslant 3$.

Remark 5.3. The map $\phi$ induces a binary operation $\oplus$ on $\mathbb{H}^{n}$ with respect to which it is a smooth quasigroup. 
The proof of the following proposition is straightforward and can be found in $[\mathbf{8}$, Theorem 10.2].

Proposition 5.4. The open disc $\mathbb{D}^{n}=\left\{\bar{x} \in \mathbb{R}^{n} \mid\|\bar{x}\|<1\right\}$ is a quasigroup with respect to the binary operation $*$ given by

$$
\bar{x} * \bar{y}=\frac{\bar{x}+\bar{y}}{1+\bar{y} \bar{x}^{t}}+\frac{1}{1+\sqrt{1-\|\bar{y}\|^{2}}} \frac{\bar{x} \bar{x}^{t} \bar{y}-\bar{y} \bar{x}^{t} \bar{x}}{1+\bar{y} \bar{x}^{t}},
$$

where $\bar{x}^{t}$ denotes the transpose of the row vector $\bar{x}=\left(x_{1}, \ldots, x_{n}\right) \in \mathbb{D}^{n}$, in which $\overline{0}$ is the identity and the inverse of $\bar{x}$ is $-\bar{x}$. Further, the Lorentz boost map $B: \mathbb{D}^{n} \longrightarrow S$ given by

$$
B(\bar{x})=\left(\begin{array}{cc}
I_{n}+\frac{\bar{x}^{t} \bar{x}}{\left(\sqrt{1-\|\bar{x}\|^{2}}\right)\left(1+\sqrt{1-\|\bar{x}\|^{2}}\right)} & \frac{\bar{x}^{t}}{\sqrt{1-\|\bar{x}\|^{2}}} \\
\frac{\sqrt{1-\|\bar{x}\|^{2}}}{\sqrt{1-\|\bar{x}\|^{2}}}
\end{array}\right)
$$

is an isomorphism from $\left(\mathbb{D}^{n}, *\right)$ to $(S, \circ)$.

Corollary 5.5. Let $\bar{x}, \bar{y} \in \mathbb{D}^{n}$. Then the following conditions are equivalent:

(i) $f^{\mathbb{D}^{n}}(\bar{x}, \bar{y})=I_{\mathbb{D}^{n}}$, the identity map on $\mathbb{D}^{n}$,

(ii) $\bar{x} * \bar{y}=\bar{y} * \bar{x}$

(iii) $\{\bar{x}, \bar{y}\}$ is linearly dependent in $\mathbb{R}^{n}$.

Proof. The equivalence of (i) and (ii) follows from Propositions 5.1 and 5.4. Next, by equation (9), it follows that $\bar{x} * \bar{y}=\bar{y} * \bar{x}$ if and only if

$$
\frac{1}{1+\sqrt{1-\|\bar{y}\|^{2}}} \frac{\bar{x} \bar{x}^{t} \bar{y}-\bar{y} \bar{x}^{t} \bar{x}}{1+\bar{y} \bar{x}^{t}}=\frac{1}{1+\sqrt{1-\|\bar{x}\|^{2}}} \frac{\bar{y} \bar{y}^{t} \bar{x}-\bar{x} \bar{y}^{t} \bar{y}}{1+\bar{x} \bar{y}^{t}} .
$$

Suppose that $\{\bar{x}, \bar{y}\}$ is linearly independent, then

$$
\frac{1}{1+\sqrt{1-\|\bar{y}\|^{2}}} \bar{x} \bar{x}^{t}=-\frac{1}{1+\sqrt{1-\|\bar{x}\|^{2}}} \bar{x} \bar{y}^{t}
$$

and

$$
-\frac{1}{1+\sqrt{1-\|\bar{y}\|^{2}}} \bar{y} \bar{x}^{t}=\frac{1}{1+\sqrt{1-\|\bar{x}\|^{2}}} \bar{y}^{t} .
$$

If $\bar{x}$ and $\bar{y}$ are orthogonal, then $\bar{x} \bar{x}^{t}=0=\bar{y} \bar{y}^{t}$ and so $\bar{x}=\overline{0}=\bar{y}$. Suppose that $\bar{x}$ and $\bar{y}$ are not orthogonal. Then

$$
\frac{\bar{x} \bar{x}^{t}}{\bar{y} \bar{x}^{t}}=\frac{\bar{x} \bar{y}^{t}}{\bar{y} \bar{y}^{t}}
$$

that is, $|\langle\bar{x}, \bar{y}\rangle|^{2}=\|\bar{x}\|^{2}\|\bar{y}\|^{2}$. This is a contradiction to the linear independence of $\{\bar{x}, \bar{y}\}$.

Corollary 5.6. A sequence $\left(\overline{x_{1}}, \overline{x_{2}}, \ldots, \overline{x_{n}}, \overline{0}, \overline{0} \ldots\right)$ is an $\mathbf{A}$-sequence in $\left(\mathbb{D}^{n}, *\right)$ if and only if $\left\{\overline{x_{i}}, \overline{x_{i+1}}\right\}$ is linearly dependent in $\mathbb{R}^{n}$ for all $i \geqslant 3$. 
Proof. The proof follows from Proposition 5.1, Proposition 5.4 and Corollary 5.5.

Corollary 5.7. Given $\bar{x} \in \mathbb{D}^{n} \backslash\{\overline{0}\}$, the subset $\mathbb{D}_{\bar{x}}=\{\alpha \bar{x}|| \alpha \mid\|\bar{x}\|<1\}$ forms a subgroup of the quasigroup $\left(\mathbb{D}^{n}, *\right)$, which is isomorphic to the additive group of real numbers, and the inclusion map $i_{\bar{x}}$ from $\left(\mathbb{D}_{\bar{x}}, G_{\mathbb{D}_{\bar{x}}}=\left\{I_{\mathbb{D}_{\bar{x}}}\right\}, \sigma^{\mathbb{D}_{\bar{x}}}, f^{\mathbb{D}_{\bar{x}}}\right)$ to

$$
\left(\mathbb{D}^{n}, G_{\mathbb{D}^{n}} \cong \mathrm{SO}(n) \times \mathrm{SO}(1), \sigma^{\mathbb{D}^{n}}, f^{\mathbb{D}^{n}}\right)
$$

is a regular c-homomorphism. Further, all maximal subgroups of $\left(\mathbb{D}^{n}, *\right)$, or equivalently of $(S, \circ)$, are isomorphic to the additive group of real numbers and any two distinct maximal subgroups intersect trivially.

Proof. By equation (9),

$$
\alpha \bar{x} * \beta \bar{x}=\frac{\alpha \bar{x}+\beta \bar{x}}{1+\alpha \beta\|\bar{x}\|^{2}} .
$$

Clearly, $\mathbb{D}_{\bar{x}}$ is an abelian group with respect to the induced binary operation $*$. Further, the map $\phi: \mathbb{R} \longrightarrow \mathbb{D}_{\bar{x}}$ given by $\phi(\alpha)=(\tanh \alpha) \frac{\bar{x}}{\|\bar{x}\|}$ is an isomorphism from the additive group $(\mathbb{R},+)$ to $\left(\mathbb{D}_{\bar{x}}, *\right)$. Since $f^{\mathbb{D}^{n}}(\alpha \bar{x}, \beta \bar{x})=I_{\mathbb{D}^{n}}$ (Corollary 5.5), it follows that the inclusion map from $\mathbb{D}_{\bar{x}}$ into $\mathbb{D}^{n}$ is a regular $c$-homomorphism. Clearly, every subgroup of $\left(\mathbb{D}^{n}, *\right)$ is contained in $\mathbb{D}_{\bar{x}}$ for some $\bar{x} \in \mathbb{D}^{n} \backslash\{\overline{0}\}$. Also, $\mathbb{D}_{\bar{x}} \neq \mathbb{D}_{\bar{y}}$ implies $\mathbb{D}_{\bar{x}} \cap \mathbb{D}_{\bar{y}}=\{\overline{0}\}$.

Theorem 5.8. There is natural homomorphism from $H^{m}\left(\mathbb{H}^{n}, M\right)$ to the group of all maps from the real projective space $\mathbb{P}^{n-1}(\mathbb{R})$ to the $m$-th cohomology group $H^{m}(\mathbb{R}, M)$, where $M$ is treated as trivial $(\mathbb{R},+)$-module and $\mathbb{H}^{n}$, the hyperbolic $n$-space with the natural right quasigroup structure described above.

Proof. Clearly, $\mathbb{D}_{\bar{x}}=\mathbb{D}_{\bar{y}}$ if and only if $[\bar{x}]=[\bar{y}]$ in $\mathbb{P}^{n-1}(\mathbb{R})$. The map $\phi \circ B \circ i_{\bar{x}}: \mathbb{D}_{\bar{x}}$ $\longrightarrow \mathbb{H}^{n}$ is a regular $c$-homomorphism. This induces a homomorphism $H^{m}\left(\phi \circ B \circ i_{\bar{x}}\right)$ from $H^{m}\left(\mathbb{H}^{n}, M\right)$ to $H^{m}\left(\mathbb{D}_{\bar{x}}, M\right) \cong H^{m}(\mathbb{R}, M)$, which is obtained by restricting a pseudo-cochain

$$
f:\left(\mathbb{H}^{n}\right)^{m} \longrightarrow M
$$

to the pseudo-cochain

$$
f \circ\left(\phi \circ B \circ i_{\bar{x}}\right)^{m}: \mathbb{D}_{\bar{x}}^{m} \longrightarrow M
$$

Remark 5.9.

(i) It can be observed that $\phi \circ B$ is the gnomonic projection of the projective disc model $\mathbb{D}^{n}$ onto $\mathbb{H}^{n}[\mathbf{1 6}$, p. 192].

(ii) Right translations in $\left(\mathbb{H}^{n}, \oplus\right)$ are the isometries of the hyperbolic space $\mathbb{H}^{n}$.

(iii) The discussions in this section hold good on any pre-Hilbert space with respect to Lorentz-Minkowski distance [2].

Next, consider the spherical $n$-space

$$
\mathbb{S}^{n}=\left\{\bar{x} \in \mathbb{R}^{n+1} \mid\|\bar{x}\|=1\right\} .
$$

It is a right quasigroup [14, Example 2.3] (see also [9]) with respect to the operation 
'o' given by

$$
\bar{x} \circ \bar{y}= \begin{cases}\left(2 P_{\frac{e_{n+1}+\bar{y}}{\left\|e_{n+1}+\bar{y}\right\|}}-I\right) J(\bar{x}), & \text { if } \bar{y} \neq-e_{n+1}, \\ -\bar{x}, & \text { if } \bar{y}=-e_{n+1},\end{cases}
$$

where $P_{\bar{a}}, \bar{a} \in \mathbb{S}^{n}$ denotes the projection of $\mathbb{R}^{n+1}$ onto $\mathbb{R} \bar{a}$ and $J$ is given by

$$
J(\bar{x})=2\left\langle\bar{x}, e_{n+1}\right\rangle e_{n+1}-\bar{x} .
$$

It can be checked that $\sigma_{\bar{x}} \mathbb{S}^{n}(h)=h$ for all $h \in G_{\mathbb{S}^{n}}$ and that $f^{\mathbb{S}^{n}}(\bar{y}, \bar{z})=I_{\mathbb{S}^{n}}$, the identity map on $\mathbb{S}^{n}$ if and only if $\left\{e_{n+1}, \bar{y}, \bar{z}\right\}$ is geodesically dependent in $\mathbb{S}^{n}$. Let $\bar{y} \in \mathbb{S}^{n}-\left\{e_{n+1},-e_{n+1}\right\}$ and consider

$$
\mathbb{S}^{1}(\bar{y})=\left\{\bar{x} \in \mathbb{S}^{n} \mid\left\{e_{n+1}, \bar{y}, \bar{x}\right\} \text { is geodesically dependent }\right\} .
$$

Then the operation ' 0 ' described by (10) induces a binary operation on $\mathbb{S}^{1}(\bar{y})$ with respect to which it is a group isomorphic to the circle group $\mathbb{S}^{1}$. Also, the inclusion map from $\mathbb{S}^{1}(\bar{y})$ to $\mathbb{S}^{n}$ is a regular $c$-homomorphism. Thus we have

Theorem 5.10. There is a natural homomorphism from the $m$-th pseudo-cohomology $H^{m}\left(\mathbb{S}^{n}, M\right)$ of $\left(\mathbb{S}^{n}, \circ\right)$ with coefficients in an abelian group $M$ to the group of all maps from the set $\mathcal{G}_{e_{n+1}}$ of all geodesics in $\mathbb{S}^{n}$ passing through $e_{n+1}$ to the $m$-th cohomology group $H^{m}\left(\mathbb{S}^{1}, M\right)$, where $M$ is treated as a trivial $\mathbb{S}^{1}$-module.

\section{Low dimensional pseudo-cohomology groups}

In this section, we interpret $H^{1}(S, M)$ and $H^{2}(S, M)$.

Proposition 6.1. Let $(S, K, \sigma, f)$ be a c-groupoid. Then

$$
H^{1}(S, K, \sigma, f, M)=H^{1}(S, M)=\operatorname{Hom}\left(M(S)_{\mathrm{ab}}, M\right) .
$$

Proof. $C^{0}(S, K, M)=\{0\}=D^{0}(S, K, M)$. Also,

$$
C^{1}(S, K, M)=\{g: S \longrightarrow M \mid g(e)=0\} .
$$

Thus

$$
\begin{aligned}
H^{1}(S, M) & =\left\{g \in C^{1} \mid \delta g=0\right\} \\
& =\left\{g \in C^{1} \mid g(e)=0 \text { and } g(y)-g(x \circ y)+g(x)=0\right\} \\
& =\operatorname{Hom}(S, M) \\
& \cong \operatorname{Hom}(M(S), M) \\
& \cong \operatorname{Hom}\left(M(S)_{\mathrm{ab}}, M\right) .
\end{aligned}
$$

This implies that

$$
H^{1}(S, M)=H^{1}\left(S, G_{S}, \sigma^{S}, f^{S}, M\right) \cong \operatorname{Hom}\left(M(S)_{\mathrm{ab}}, M\right) .
$$

Example 6.2. Let $(G, \cdot)$ be a group. Define a binary operation $\circ$ on $G$ by $x \circ y=$ $y^{-1} \cdot x \cdot y^{2}$. Then it can be seen that $(G, \circ)$ is a right quasigroup with an identity whose group torsion is $[G, G] /([G, G] \cap Z(G))$. As described in Section $3, M(G)$ is a group together with a map $\alpha: G \longrightarrow M(G)$ satisfying $\alpha\left(y^{-1} x y^{2}\right)=\alpha(x) \alpha(y)$ such 
that given any group $K$ and a map $\beta: G \longrightarrow K$ satisfying $\beta\left(y^{-1} x y^{2}\right)=\beta(x) \beta(y)$, there is a unique homomorphism $\eta: M(G) \longrightarrow K$ such that $\beta=\eta \circ \alpha$. Consider the quotient map $\nu: G \longrightarrow G / G_{3}$, where $G_{3}=[G,[G, G]]$ is the third term of the lower central series of $G$. It can be observed that $G / G_{3}$ is also a group with respect to the binary operation $\circ$ given by $\left(x G_{3}\right) \circ\left(y G_{3}\right)=\left(y^{-1} \cdot x \cdot y^{2}\right) G_{3}$ and the map $\nu$ satisfies $\nu\left(y^{-1} x y^{2}\right)=\nu(x) \circ \nu(y)$. From the universal property of $M(G)$, we have a unique surjective map $\eta: M(G) \longrightarrow G / G_{3}$ given by $\eta(\alpha(g))=g G_{3}$, which is a homomorphism from the group $M(G)$ to the group $\left(G / G_{3}, \circ\right)$. It follows that it is an isomorphism. Thus we have a homomorphism

$$
\chi: \operatorname{Hom}\left(\left(G / G_{3}\right)_{\mathrm{ab}}, M\right)=\operatorname{Hom}\left(G_{\mathrm{ab}}, M\right) \longrightarrow \operatorname{Hom}\left(M(G)_{\mathrm{ab}}, M\right)=H^{1}(G, M)
$$

given by $\chi(f)=\eta_{\mathrm{ab}} \circ f$. It also follows that $\chi$ is an isomorphism.

Definition 6.3. Let $(S, \circ)$ be a right quasigroup and $M$ be an abelian group. A map $g: S^{m}=\underbrace{S \times S \times \cdots \times S}_{m \text { copies }} \longrightarrow M$ is called a $m$-linear map if

$$
\begin{aligned}
g\left(x_{1}, \ldots, x_{i-1}, x_{i} \circ x_{i+1}, x_{i+2}, \ldots, x_{m+1}\right)= & g\left(x_{1}, \ldots, x_{i-1}, x_{i}, x_{i+2}, \ldots, x_{m+1}\right) \\
& +g\left(x_{1}, \ldots, x_{i-1}, x_{i+1}, x_{i+2}, \ldots, x_{m+1}\right)
\end{aligned}
$$

for all $x_{1}, x_{2}, \ldots, x_{m+1} \in S$ and for $i,(1 \leqslant i \leqslant m)$.

Proposition 6.4. Let $(S, \circ)$ be a right quasigroup with identity and $M$ be an abelian group. Let $g: S^{m} \longrightarrow M$ be a m-linear map. Then

$$
\begin{aligned}
g\left(x_{1}, \ldots, x_{i-2}, x_{i-1} \theta f^{s}\left(x_{i}, x_{i+1}\right)\right. & \left., x_{i}, \ldots, x_{m}\right) \\
& =g\left(x_{1}, \ldots, x_{i-1}, x_{i}, x_{i+1}, \ldots, x_{m}\right),(2 \leqslant i \leqslant m-1)
\end{aligned}
$$

and it induces a m-linear map $\bar{g}: M(S)^{m} \longrightarrow M$ given by

$$
\bar{g}\left(\overline{x_{1}}, \overline{x_{2}}, \ldots, \overline{x_{m}}\right)=g\left(x_{1}, x_{2}, \ldots x_{m}\right)
$$

for all $x_{1}, x_{2}, \ldots x_{m} \in S$, where $\overline{x_{i}}$ denotes the class of $x_{i}$ in $M(S)$.

Proof. Fix a $z=\left(z_{1}, z_{2}, \ldots, z_{i-1}, z_{i+1}, \ldots, z_{m}\right) \in S^{m-1}$. Define a map $g_{z}: S \longrightarrow M$ by $g_{z}(x)=g\left(z_{1}, z_{2}, \ldots, z_{i-1}, x, z_{i+1}, \ldots, z_{m}\right)$. Then $g_{z}$ is a homomorphism from $S$ to $M$. This induces a homomorphism from $M(S)$ to $M$. Thus $g_{z}\left(x \theta f^{s}(u, v)\right)=g_{z}(x)$ for all $z$ in $S^{m-1}$ and $u, v$ in $S$. Hence $g$ induces a $m$-linear map $\bar{g}: M(S)^{m} \longrightarrow M$ as desired.

Proposition 6.5. Let $(S, K, \sigma, f)$ be a c-groupoid and $M$ be an abelian group. Let $g: S^{m} \longrightarrow M$ be a m-linear map. Then $g \in \operatorname{Ker} \delta^{m}$.

Proof. The proof follows from the definition of $\delta$ and the Proposition 6.4.

Consider the particular case $m=2$ and let us denote the set of all bilinear maps from $S \times S$ to $M$ by

$$
\operatorname{Bil}(S, M) \cong \operatorname{Bil}(M(S), M)
$$

One observes that $\operatorname{Bil}(S, M) \subseteq \operatorname{Ker} \delta^{2}$ (in general equality need not hold). It can be seen that a map $h=\delta g: S \times S \longrightarrow M$ given by $h(x, y)=g(y)-g(x \circ y)+g(x)$, 
where $g$ is map from $S$ to $M$ with $g(x \theta f(u, v))=g(x)$ for all $x, u, v \in S$, is a bilinear map if and only if

$$
g((x \circ y) \circ z)=g(x \circ y)+g(y \circ z)+g(x \circ z)-g(x)-g(y)-g(z)
$$

for all $x, y, z \in S$. Let us call such a map $g$ to be a quasi-homomorphism. It is easily observed that every homomorphism is a quasi-homomorphism. Let us denote the set of all quasi-homomorphism from $S$ to $M$ by $Q H(S, M)$. This is a group under obvious addition and $\operatorname{Hom}(S, M)=H^{1}(S, M)$ is a subgroup of $Q H(S, M)$. The pseudocoboundary map $\delta$ induces a homomorphism from $Q H(S, M)$ to the group $\operatorname{Bil}(S, M)$. The above discussion summarizes into the following proposition.

Proposition 6.6. We have the following 5-term exact sequence:

$$
0 \longrightarrow H^{1}(S, M) \stackrel{i}{\longrightarrow} Q H(S, M) \stackrel{\delta}{\longrightarrow} \operatorname{Bil}(S, M) \stackrel{j}{\longrightarrow} H^{2}(S, M)
$$

where $i$ is the inclusion map and $j(h)=h+B^{2}(S, M)$ for all $h \in \operatorname{Bil}(S, M)$.

Proof. Clearly we see that $i$ is injective and $\delta g=0$ if and only if $g \in \operatorname{Hom}(S, M)$. Also, $h \in B^{2}(S, M)$ for $h \in \operatorname{Bil}(S, M)$ if and only if $h=\delta g$ for some $g \in Q H(S, M)$.

\section{Pseudo-cohomology of group extensions}

We now try to introduce pseudo-cohomology functors from the category $\mathcal{E}_{H}$ of general extensions of $H$ to the category of abelian groups. Let $\left\{\left(X_{\alpha}, Y_{\alpha}\right), d_{\alpha}\right\}_{\alpha \in \Lambda}$ be a family of pseudo-chain complexes. Then $\left\{\left(\underset{\alpha \in \Lambda}{\oplus} X_{\alpha}, \underset{\alpha \in \Lambda}{\oplus} Y_{\alpha}\right), \underset{\alpha \in \Lambda}{\oplus} d_{\alpha}\right\}$ is a pseudochain complex called the direct sum of the family. Clearly, $H_{n}$ commutes with direct sum.

If $G$ is an extension of $H$ and $\mathcal{T}(G, H)$ the set of all right transversals of $H$ in $G$, then each $S \in \mathcal{T}(G, H)$ determines a $c$-groupoid, which, in short, will be denoted by $(S, H)$. Define

$$
P^{*}(G, H, M)=\bigoplus_{S \in \mathcal{T}(G, H)} P^{*}(S, H, M)
$$

Further,

$$
H^{n}\left(P^{*}(G, H, M)\right)=\bigoplus_{S \in \mathcal{T}(G, H)} H^{n}(S, H, M)
$$

is called the $n$-th pseudo-cohomology group of the extension $(G, K)$ with coefficients in $M$ and is denoted by $H^{n}(G, H, M)$.

We show that $P^{*}$ defines a contravariant functor from the category $\mathcal{E}_{H}$ to the category of pseudo-cochain complexes of abelian groups.

Let $G_{1}$ and $G_{2}$ be extensions of $H$ and $q$ a $H$-homomorphism from $G_{1}$ to $G_{2}$, which fixes members of $H$. If $\mathcal{T}_{1}\left(G_{1}, H\right)$ and $\mathcal{T}_{2}\left(G_{2}, H\right)$ are the corresponding sets of right transversals, then one can easily check that for each $S_{2} \in \mathcal{T}_{2}\left(G_{2}, H\right), q^{-1}\left(S_{2}\right)=S_{1}$ is a member of $\mathcal{T}_{1}\left(G_{1}, H\right)$ such that $\left(\left.q\right|_{S_{1}},\left.q\right|_{H}\right)$ is a regular $c$-homomorphism from $\left(S_{1}, H\right)$ to $\left(S_{2}, H\right)$. This gives us a pseudo-cochain transformation from $P^{*}\left(S_{2}, H, M\right)$ to $P^{*}\left(S_{1}, H, M\right)$, which in turn defines a unique pseudo-chain transformation $P^{*}(q)$ 
from $P^{*}\left(G_{2}, H, M\right)$ to $P^{*}\left(G_{1}, H, M\right)$. It is easy to see now that $P^{*}$ is a contravariant functor. For each $n, H^{n}$, therefore is a contravariant functor from $\mathcal{E}_{H}$ to the category of abelian groups.

The one and two dimensional pseudo-cohomology groups have easy interpretations in many cases. For example, if $G$ is a Schreier extension of $H$, then each transversal $S$ is a group isomorphic to $G / H$; hence

$$
H^{1}(G, H, M) \cong \bigoplus m^{n-1} \operatorname{Hom}(G / H, M) \cong \bigoplus m^{n-1} \operatorname{Hom}\left((G / H)_{\mathrm{ab}}, M\right)
$$

and

$$
H^{2}(G, H, M) \cong \bigoplus^{m^{n-1}} H_{\phi}^{2}(G / H, M)
$$

where $m$ is the cardinality of $H, n$ is that of $G / H$ and $\phi$ is the trivial homomorphism from $G / H$ to Aut $M$. Also, if $G$ is an extension of $H$ such that $G=H K$ (where $K$ is a subgroup of $G) \Rightarrow K=G$, then

$$
H^{1}(G, H, M) \cong \bigoplus \operatorname{Hom}\left(G / H^{G}, M\right)
$$

where $H^{G}$ is the normal closure of $H$ (Proposition 6.1).

To study higher dimensional pseudo-cohomology groups is, of course, difficult. We try to compute $H^{n}(G, H, M)$ for the case when $H$ is a direct factor of $G$.

Let $H$ and $K$ be two groups and $g$ an identity preserving map from $K$ to $H$ and $X^{n}$, the set of all maps from $K^{n}$ to $M$, where $M$ is an abelian group. Define a map $\delta^{n}: X^{n} \longrightarrow X^{n+1}$ as follows:

$$
\begin{aligned}
& \delta^{n} f\left(x_{1}, x_{2}, \ldots, x_{n+1}\right)
\end{aligned}
$$

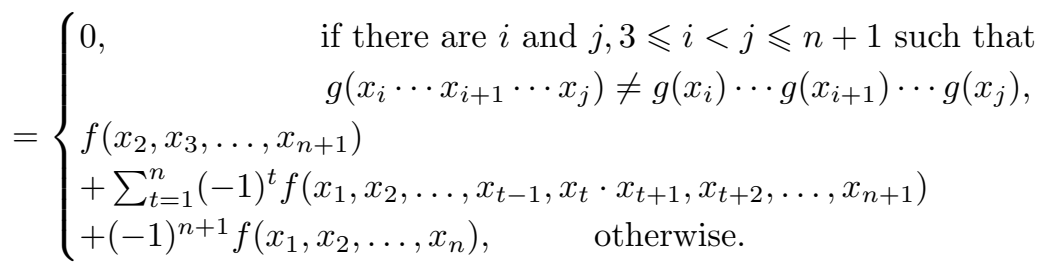

Then $\left\{X^{n}, \delta^{n}\right\}$ is a cochain complex. The $n$-th homology of this cochain complex will be denoted by $H^{n}(K, H, g, M)$. If $g$ turns out to be a homomorphism, then $H^{n}(K, H, g, M)=H^{n}(K, M) \cong \operatorname{Ext}_{\mathbb{Z}(K)}^{n}(\mathbb{Z}, M)$, the $n$-dimensional cohomology group of $K$ with coefficients in $M$ considered as a trivial $K$-module.

Theorem 7.1. Let $H$ be a direct factor of $G$. Then

$$
H^{n}(G, H, M)=\bigoplus_{g \in A} H^{n}(G / H, H, g, M),
$$

where $A$ is the set of identity preserving maps from $G / H$ to $H$.

Proof. Suppose that $G=H \times K$. Then each identity preserving map $g$ from $K$ to $H$ uniquely determines a right transversal $\{g(x) x \mid x \in K\}$. Further, these are the only right transversals. Let $(S, H, \sigma, f)$ denote the $c$-groupoid determined by 
$\{g(x) x \mid x \in K\}$. Since $(S, H, \sigma, f)$ is a factor system, each $\sigma_{g(x) x}$ is an automorphism of $H$. Therefore, $\sigma_{g(x) x}(f(g(y) y, g(z) z))=1$ if and only if

$$
(f(g(y) y, g(z) z))=1 .
$$

Now $g(x) x \cdot g(y) y=g(x) g(y) x y=g(x) g(y) g(x y)^{-1}(g(x y) x y)$, for $H$ is a direct factor. This shows that $f(g(x) x, g(y) y)=g(x) g(y) g(x y)^{-1}$. The result now follows from the definitions of $H^{n}(K, H, g, M)$ and $H^{n}(G, H, M)$.

Corollary 7.2. Let $H$ be a direct factor of $G$. Then

$$
H^{n}(G, H, M)=\stackrel{r}{\oplus}\left(\operatorname{Ext}_{\mathbb{Z}(G / H)}^{n}(\mathbb{Z}, M)\right)\left(\oplus H^{n}(G / H, H, g, M)\right),
$$

where $r$ is the cardinality of $\operatorname{Hom}(G / H, H)$, and the second part of the summand is taken over all identity preserving maps from $G / H$ to $H$ which are not homomorphisms.

Corollary 7.3. If $G$ is an split extension of $H$, then $\operatorname{Ext}_{\mathbb{Z}(G / H)}^{n}(\mathbb{Z}, M)$, where $M$ is trivial $G / H$ module, is a direct summand of $H^{n}(G, H, M)$.

Let $G$ be a finite group, which is an extension of $H$, and $K$ be another group. Con-

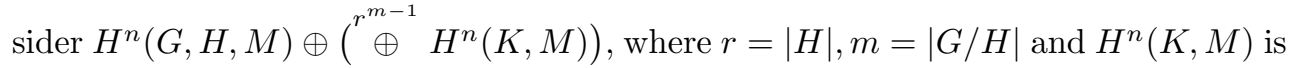
the $n$-th cohomology of $K$ with coefficients in $M$, which is considered as a trivial $K$ module. Now we define a homomorphism $\tau$ from $H^{n}(G, H, M) \oplus\left(\stackrel{r^{m-1}}{\oplus} H^{n}(K, M)\right)$ to $H^{n}(G \times K, H, M)$ as follows: Let $S$ be a right transversal of $H$ in $G$. Then $S K=$ $\{x k \mid x \in S, k \in K\}$ is a right transversal of $G \times K$ modulo $H$. Let $f: S^{n} \longrightarrow M$ be a $n$-pseudo-cocycle in $P^{*}(S, H, M)$ and $g: K^{n} \longrightarrow M$ be a $n$-cocycle in $P^{*}(K, M)$. Define $f \times g:(S K)^{n} \longrightarrow M$ as

$$
(f \times g)\left(a_{1} b_{1}, a_{2} b_{2}, \ldots, a_{n} b_{n}\right)=f\left(a_{1}, a_{2}, \ldots, a_{n}\right)+g\left(b_{1}, b_{2}, \ldots, b_{n}\right) .
$$

From routine computation, we see that $f \times g$ is a $n$-pseudo-cocycle in $P^{*}(S K, H, M)$ and if $f$ is a $n$-pseudo-coboundary and $g$ is a $n$-coboundary, then $f \times g$ is a $n$ pseudo-coboundary in $P^{*}(S K, H, M)$. Therefore, this induces a homomorphism from $H^{n}(S, H, M) \oplus H^{n}(K, M)$ to $H^{n}(S K, H, M)$ and hence a homomorphism $\tau$ from $H^{n}(G, H, M) \oplus\left(\stackrel{r^{m-1}}{\oplus} H^{n}(K, M)\right)$ to $H^{n}(G \times K, H, M)$. This homomorphism $\tau$ may be of help in getting the relationship between the pseudo-cohomology groups of two extensions, namely almost equivalent extensions and related extensions.

\section{Pseudo-homology of $c$-groupoids and right quasigroups}

Let $(S, H, \sigma, f)$ be a $c$-groupoid. Define addition and multiplication in $\mathbb{Z}(S)=$ $\{r: S \longrightarrow \mathbb{Z} \mid r(x)=0$ for all but finitely many $x$ in $S\}$ as follows:

$$
(r+s)(s)=r(x)+s(x) \quad \text { and } \quad(r . s)(x)=\sum_{y \circ z=x} r(y) s(z) .
$$

Then $\mathbb{Z}(S)$ is a ring (not necessarily associative) called the integral right quasigroup ring. 
Let $X_{n}$ denote the free $\mathbb{Z}(S)$-module generated over the free base of ordered $n$ tuples $\left[x_{1}, x_{2}, \ldots, x_{n}\right]$ of elements of $S$, where no $x_{i}$ is $e$. One may describe $X_{n}$ as a free abelian group with a basis consisting of elements of the type $x\left[x_{1}, x_{2}, \ldots, x_{n}\right]$, where no $x_{i}$ is $e$. Here $x \cdot\left(y \cdot\left[x_{1}, x_{2}, \ldots, x_{n}\right]\right)$ has been defined to be $(x \circ y)\left[x_{1}, x_{2}, \ldots, x_{n}\right]$. For convenience, let us put $\left[x_{1}, x_{2}, \ldots, x_{n}\right]=0$, if some $x_{i}=e$.

Let $Y_{n}$ be the submodule of $X_{n}$ generated by

$$
\left\{\left[x_{1}, x_{2}, \ldots, x_{n}\right] \mid x_{1} \theta f\left(x_{2}, x_{3}\right)=x_{1} \text { and } x_{n-2} \theta f\left(x_{n-1}, x_{n}\right)=x_{n-2}, n \geqslant 3\right\} .
$$

Define a map $d_{n}: X_{n} \longrightarrow X_{n-1}$ as follows:

$$
\begin{aligned}
d_{n} & \left(\left[x_{1}, x_{2}, \ldots, x_{n}\right]\right) \\
& =\left\{\begin{array}{l}
0, \quad \text { if }\left(x_{1}, x_{2}, \ldots, x_{n}, e, e \ldots\right) \text { is not an A-sequence, } \\
x_{1}\left[x_{2}, x_{3}, \ldots, x_{n}\right] \\
+\sum_{t=1}^{n-1}(-1)^{t}\left[x_{1}, x_{2}, \ldots, x_{t-1} \theta f\left(x_{t}, x_{t+1}\right), x_{t} \circ x_{t+1}, x_{t+2}, \ldots, x_{n}\right] \\
+(-1)^{n}\left[x_{1}, x_{2}, \ldots, x_{n-1}\right],
\end{array}\right.
\end{aligned}
$$

Clearly, if some $x_{i}=e$, then $d_{n}\left(\left[x_{1}, x_{2}, \ldots, x_{n}\right]\right)=0$. Now, as in Theorem 4.4, one can easily show, after a little computation, that $(X, Y)=\left\{\left(X_{n}, Y_{n}\right), d_{n}\right\}$ is a pseudochain complex. By definition, $X_{0}=Y_{0}$ is a $\mathbb{Z}(S)$-module over one generator. Define $\epsilon$, the augmentation map from $\mathbb{Z}(S)$ to $\mathbb{Z}$ as $\epsilon\left(\sum \alpha_{i} x_{i}\right)=\sum \alpha_{i}$. Clearly, $\epsilon$ is a $\mathbb{Z}(S)$ module homomorphism, where $\mathbb{Z}$ is the trivial $\mathbb{Z}(S)$-module. Thus we have a positive pseudo-chain complex

$$
\cdots \longrightarrow\left(X_{n}, Y_{n}\right) \longrightarrow\left(X_{n-1}, Y_{n-1}\right) \cdots \longrightarrow\left(X_{0}, Y_{0}\right) \longrightarrow \mathbb{Z} \longrightarrow 0,
$$

where $X_{0}=Y_{0}=\mathbb{Z}(S)$.

Let $M$ be a $\mathbb{Z}(S)$-module. Denote $(X, Y) \otimes M$ by $P_{*}(S, H, M)$. Further, $P_{*}$ defines a covariant functor from $\mathcal{E}_{H}$ to the category of chain complexes as

$$
P_{*}((G, H), M)=\bigoplus_{S \in \mathcal{T}(G, H)} P_{*}(S, H, M) .
$$

The $n$-th pseudo-homology $H_{n}\left(P_{*}((G, H), M)\right)$, denoted by $H_{n}(G, H, M)$, is called the $n$-dimensional pseudo-homology of the extension $G$ of $H$ with coefficients in $M$. For each $n, H_{n}$ is a covariant functor from $\mathcal{E}_{H}$ to the category of abelian groups.

As in the case of pseudo-cohomology, we can interpret $H_{n}(G, H, M)$ for the case when $H$ is a direct factor of $G$.

Let $K$ be another group and $g$ be an identity preserving map from $K$ to $H$. Define $X_{n}$ as a free $\mathbb{Z}(K)$-module generated by the elements of the type $\left[x_{1}, x_{2}, \ldots, x_{n}\right]$, where $\left[x_{1}, x_{2}, \ldots, x_{n}\right]=0$ if any $x_{i}=1$. Define $d_{n}: X_{n} \longrightarrow X_{n-1}$ as

$$
d_{n}\left[x_{1}, x_{2}, \ldots, x_{n}\right]=\left\{\begin{array}{l}
0, \quad \begin{array}{l}
\text { if there are } i \text { and } j, 3 \leqslant i<j \leqslant n \text { such that } \\
g\left(x_{i} \cdots x_{i+1} \cdots x_{j}\right) \neq g\left(x_{i}\right) \cdots g\left(x_{i+1}\right) \cdots g\left(x_{j}\right), \\
x_{1}\left[x_{2}, x_{3}, \ldots, x_{n}\right] \\
+\sum_{1}^{n-1}(-1)^{t}\left[x_{1}, x_{2}, \ldots, x_{t-1}, x_{t} \cdot x_{t+1}, x_{t+2}, \ldots, x_{n}\right] \\
+(-1)^{n}\left[x_{1}, x_{2}, \ldots, x_{n-1}\right],
\end{array} \quad \text { otherwise. }
\end{array}\right.
$$

Then $\left\{\left(X_{n}, d_{n}\right)\right\}$ is a chain complex. Define $H_{n}\left(\left(X_{n}, d_{n}\right) \otimes M\right)=H_{n}(K, H, g, M)$. 
Theorem 8.1. Let $H$ be a direct factor of $G$. Then

$$
H_{n}(G, H, M)=\underset{g}{\oplus} H_{n}(G / H, H, g, M),
$$

the direct sum taken over all identity preserving maps $g$ from $G / H$ to $H$.

The proof is similar to that of Theorem 7.1.

Corollary 8.2. Under the hypothesis of Theorem 8.1,

$$
H_{n}(G, H, M)=\left(\stackrel{\alpha}{\oplus} \operatorname{Tor}_{n}^{\mathbb{Z}(G / H)}(\mathbb{Z}, M)\right) \oplus\left(\underset{g}{\oplus} H_{n}(G / H, H, g, M)\right),
$$

where $\alpha$ is the cardinality of $\operatorname{Hom}(G / H, H)$, and the second summation is over all identity preserving maps $g$ from $G / H$ to $H$ which are not homomorphisms.

\section{Some problems}

Suppose that all transversals of a subgroup $H$ in a group $G$ are isomorphic as right loops. Then $H^{n}(S, M) \cong H^{n}(T, M)$ for every pair of transversals $S$ and $T$ of a subgroup $H$ in $G$. What can we say about the converse? Indeed, if $G$ is finite and all transversals of $H$ in $G$ are isomorphic, then $H$ is a normal subgroup of $G[\mathbf{1 3}$, Main Theorem]. Is this statement also valid if we simply demand that $H^{n}(S, M) \cong$ $H^{n}(T, M)$ for every pair of transversals $S$ and $T$ of a subgroup $H$ in a finite group $G$ ? In particular, one may discuss the subgroup $H$ of $G$ for which

$$
H^{1}(S, M) \cong \operatorname{Hom}\left(M(S)_{\mathrm{ab}}, M\right) \cong H^{1}(T, M) \cong \operatorname{Hom}\left(M(T)_{\mathrm{ab}}, M\right)
$$

for all $M$ and for all pairs $S$ and $T$ of transversals of $H$ in $G$. Clearly, then $M(S)_{\mathrm{ab}} \cong$ $M(T)_{\text {ab }}$ for all pairs $S$ and $T$ of transversals of $H$ in $G$. We note that if $H$ is a subgroup of a finite simple group having no complement, then $M(S)=\{e\}$ for all transversals $S$ of $H$ in $G$ [12]. For a finite solvable group it is known $([\mathbf{1 2}])$ that $M(S) \cong M(T)$ for all pairs of transversals $S$ and $T$ of an arbitrary subgroup $H$ of $G$, if and only if $G$ is a Dedekind group. What can we say about finite group for which given any subgroup $H$ of $G$ and transversals $S$ and $T$ of $H$ in $G, M(S)_{\mathrm{ab}} \cong M(T)_{\mathrm{ab}}$ (equivalently, $\left.H^{1}(S, M) \cong H^{1}(T, M)\right)$ ? In particular, what can we say about a finite group $G$ for which $G=H K \Longrightarrow K / K^{\prime}[K \cap H]^{K} \cong G / G^{\prime} H^{G}$ ? We may further ask to characterize groups $G$ for which given a subgroup $H$ of $G$ and right transversals $S$ and $T$ of $H$ in $G, Q H(S, M) \cong Q H(T, M)\left(\operatorname{Bil}(S, M) \cong \operatorname{Bil}(T, M), H^{2}(S, M) \cong H^{2}(T, M)\right)$ for all abelian groups $M$.

Several other problems arise out of the discussions of the paper. We also need to develop some methods to compute pseudo-cohomology and pseudo-homology groups of some important types of extensions.

\section{References}

[1] R. Baer, Klassifikation der gruppenerweiterungen, J. Reine Angew. Math. 187 (1949), 75-94.

[2] W. Benz, Lorentz-Minkowski distances in Hilbert spaces, Geom. Dedicata 81 (2000), nos. 1-3, 219-230. 
[3] S. Eilenberg and S. MacLane, Group extensions and homology, Ann. of Math. (2) 43 (1942), no. 4, 757-831.

[4] S. Eilenberg and S. MacLane, Cohomology theory in abstract groups I, Ann. of Math. (2) 48 (1947), no. 1, 51-78.

[5] S. Eilenberg and S. MacLane, Cohomology theory in abstract groups II, Ann. of Math. (2) 48 (1947), no. 2, 326-341.

[6] S. Eilenberg and S. MacLane, Algebraic cohomology groups and loops, Duke Math. J. 14 (1947), no. 2, 435-463.

[7] K.W. Johnson and C.R. Leedham-Green, Loop cohomology, Czechoslovak Math. J. 40(115) (1990), no. 2, 182-194.

[8] H. Kiechle, Theory of K-loops, Lecture Notes in Math. 1778, Springer-Verlag, New York, 2002.

[9] M.K. Kinyon, Global left loop structures on spheres, Comment. Math Univ. Carolin. 41 (2000), no. 2, 325-346.

[10] J.P. Lafuente, Parametrizing group extension loops, Comm. Algebra 28 (2000), no. $6,2783-2800$.

[11] R. Lal, Transversals in groups, J. Algebra 181 (1996), no. 1, 70-81.

[12] R. Lal, Some problems on Dedekind-type groups, J. Algebra 181 (1996), no. 1, 223-234.

[13] R. Lal and R.P. Shukla, Perfectly stable subgroups of finite groups, Comm. Algebra 24 (1996), no. 2, 643-657.

[14] R. Lal and R.P. Shukla, Transversals in non-discrete groups, Proc. Indian Acad. Sci. (Math. Sci.) $\mathbf{1 1 5}$ (2005), no. 4, 429-435.

[15] S. MacLane, Homology, Springer-Verlag, New York, 1963.

[16] J.G. Ratcliffe, Foundations of hyperbolic manifolds, Grad. Texts in Math. 149, Springer-Verlag, New York, 1994.

[17] J.D.H. Smith, An introduction to quasigroups and their representations, Stud. Adv. Math., Chapman \& Hall/CRC, Boca Raton, FL, 2007.

Ramji Lal mathrjl@gmail.com

Department of Mathematics, University of Allahabad, Allahabad 211002, India

B.K. Sharma bksharma@allduniv.ac.in

Department of Mathematics, University of Allahabad, Allahabad 211002, India 